\section{Upper airway obstruction due to inoperable intrathoracic goitre treated by tracheal endoprosthesis}

\author{
M Noppen, M Meysman, E Dhondt, \\ L Gepts, B Velkeniers, L Vanhaelst, \\ W Vincken
}

\begin{abstract}
Thyroidectomy is the treatment of choice in patients with thyroid enlargement complicated by compression or displacement of the trachea because of the risk of complete airway obstruction due to sudden enlargement of the goitre by, for example, haemorrhage. In patients who are medically inoperable an endoscopically inserted tracheal endoprosthesis may provide longstanding airway patency, as reported here.
\end{abstract}

(Thorax 1994;49:1034-1036)

Insertion of tracheal, bronchial, or tracheobronchial stents can offer excellent palliation in patients with central airway obstruction who are not amenable to surgery. ${ }^{12}$ Tracheal or bronchial malignant processes with extrinsic airway compression constitute the major indication for airway stenting, although longstanding success has been achieved in patients with benign tracheostenosis following intubation. $^{3}$ We therefore attempted tracheal stenting in two patients with acute upper airway obstruction due to non-malignant intrathoracic goitre in whom thyroidectomy was contraindicated.

\section{Case 1}

A 90 year old woman was surgically treated for a left femoral fracture in a general hospital under locoregional anaesthesia. Postoperatively she developed acute severe inspiratory and expiratory dyspnoea. She was known to have a large intrathoracic goitre, causing tracheal displacement and narrowing, which was responsible for her acute respiratory distress.

The patient, in otherwise good general condition, was transferred to our department for endoscopic treatment. On examination she was conscious, tachypnoeic ( 25 breaths/min) and anxious. Both inspiration and expiration were
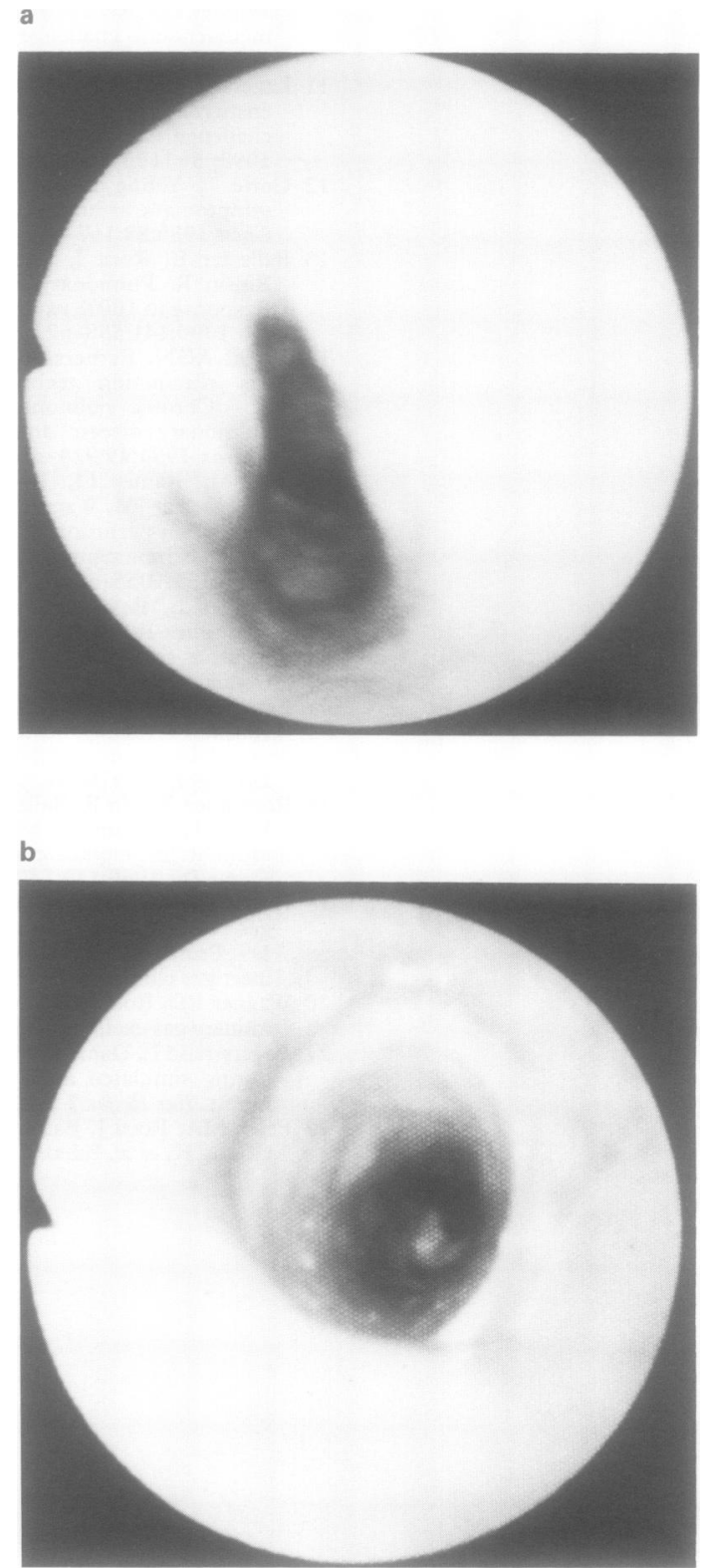

Figure 1 Fibreoptic view of the tracheal stenosis in case 1 (a) before and (b) after insertion of the tracheal stent.

shallow and accompanied by a permanent wheeze. Physical examination was otherwise normal except for the scar of the recent hip intervention. There were no signs of venous thrombosis.

A radiograph of the trachea showed severe displacement and narrowing by the intrathoracic goitre. After topical anaesthesia of the oropharynx ( $10 \%$ lignocaine spray) and vocal cords ( $2 \%$ lignocaine, $5 \mathrm{ml}$ ) a fibreoptic bronchoscopy was performed which showed severe compression of the trachea from both sides (residual lumen $6 \mathrm{~mm}$ diameter) over a length of $4.5 \mathrm{~cm}$ (fig 1a). The distal airways (which were quickly inspected) appeared normal.

Since thyroidectomy was considered to be contraindicated in this elderly patient, endoscopic placement of a tracheal 
endoprosthesis was attempted. Under light general anaesthesia with intravenous propofol rigid bronchoscopy (Storz, Germany) was performed. Ventilation and oxygenation were maintained with high frequency jet ventilation delivered via a side port of the bronchoscope. A silicone Dumon tracheal endoprosthesis (Endoxane $T D$, diameter $12 \mathrm{~mm}$, length $4 \mathrm{~cm}$; Cometh Laboratoire, Marseille, France) was longitudinally folded and pushed through the siliconised lumen of the properly positioned bronchoscope through the stenosis. Under direct vision the prosthesis was unfolded with forceps. After ensuring proper placement and fixation of the prosthesis, the bronchoscope was removed. The entire procedure lasted 20 minutes and the postoperative course was uneventful. Follow up fibreoptic bronchoscopy the next day showed good airway patency (fig 1b); the patient was discharged the same day.

There was no recurrence of upper airway obstruction four months after insertion of the stent.

a

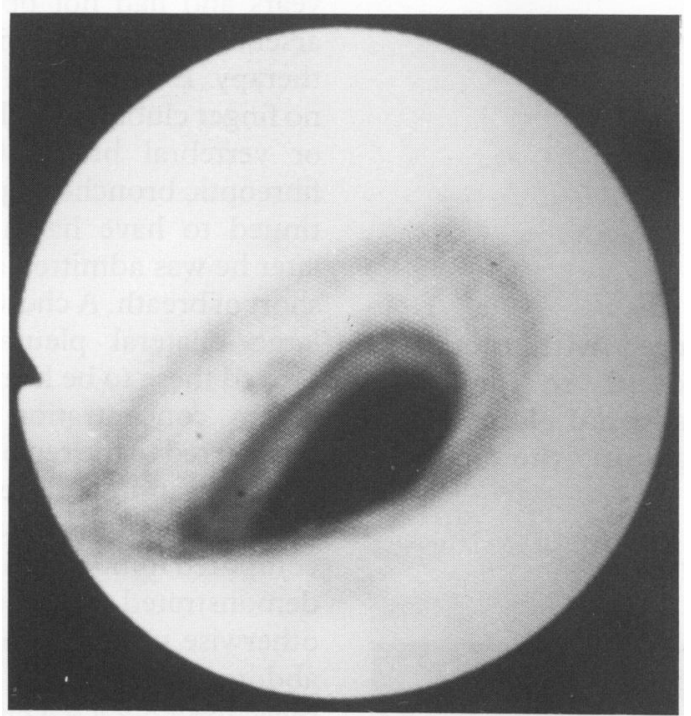

b

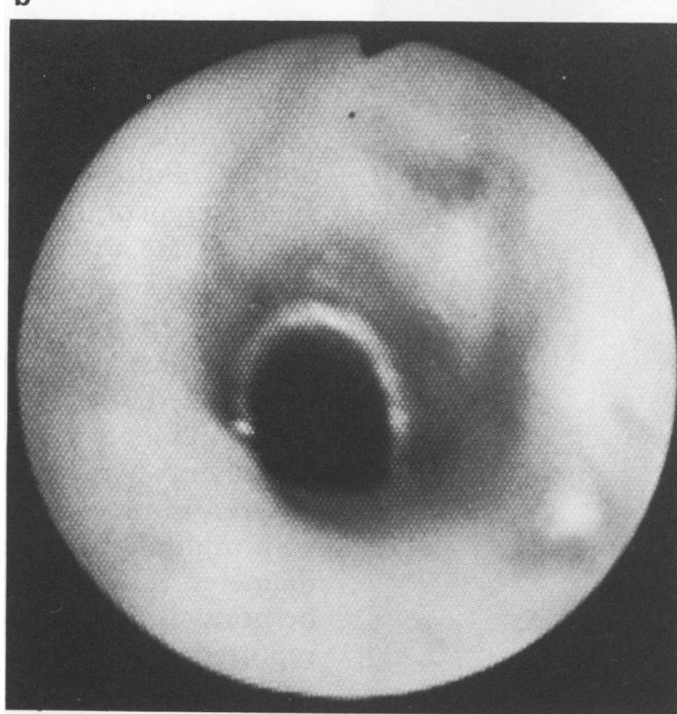

Figure 2 Fibreoptic view of the tracheal stenosis in case 2 (a) before and (b) after insertion of the stent.

\section{Case 2}

An 89 year old woman with a large multinodular goitre presented with rapidly progressive dyspnoea, stridor, and dysphagia due to a sudden increase in size of her goitre following an intrathyroid haemorrhage. On examination she was tachypnoeic with stridors at rest; vital parameters were normal. Radiographic examination and tomographic scanning showed narrowing and displacement of the trachea. Spirometry and a flow-volume loop was suggestive of a fixed mixed intrathoracic and extrathoracic upper airway obstruction. Fibreoptic bronchoscopy showed bilateral wall narrowing and tortuous displacement of the trachea over $4 \mathrm{~cm}$; the diameter of the tracheal lumen was about $5 \mathrm{~mm}$ (fig 2a).

Surgery was considered to be contraindicated. A silicone Dumon tracheal endoprosthesis (Endoxane TD, diameter $14 \mathrm{~mm}$, length $4 \mathrm{~cm}$ ) was inserted into the stenosis via a rigid bronchoscope under general anaesthesia using high frequency jet ventilation (fig 2b). The immediate postoperative course was uneventful with complete disappearance of dyspnoea and stridor. However, 48 hours after the procedure acute stridor recurred; urgent bronchoscopy showed a stent obstruction due to fibropurulent debris. After bronchial toilet, oedematous tracheal mucosa was visualised just distal to the stent. The stent was removed and the mucosal obstruction was vapourised with Nd:YAG laser photodestruction. The stent was reinserted and stable airway patency and respiration were restored. Daily inhalations of a mucolytic agent (mesna) were prescribed.

Over the following days there were occasional short episodes of dyspnoea, probably due to partial stent obstruction by secretions in the very tortuous trachea. A reintervention one month later was therefore performed with removal of the first stent which was replaced by a longer $(5 \mathrm{~cm})$ model.

Thereafter the patient was completely asymptomatic. She died two months later due to an acute myocardial infarction. At necropsy the stent was completely patent.

\section{Discussion}

Thyroidectomy is the treatment of choice for most intrathoracic goitres because of the risk of intrathyroideal haemorrhage, especially when symptoms or signs of treacheal obstruction are present. ${ }^{4-6}$ Surgery is recommended when peak expiratory flows decrease to $<1001 / \mathrm{min}^{7}$ In high risk patients, or those in whom surgery is contraindicated, alternative means of airway protection must be sought.

In patients with intrinsic or extrinsic tracheal obstruction who are not amenable to surgery, placement of tracheal endoprostheses (stents) can offer palliation. ${ }^{12}$ Of the various stents commercially available, the silicone Dumon ${ }^{2}$ and the expandable metal Gianturco $\mathrm{Z}$ stent ${ }^{8}$ are the most widely used. Both are easily inserted, are efficacious in stenoses up to $5 \mathrm{~cm}$ in length, and are well tolerated. Advantages of the silicone Dumon stents are the possibility of adjustment and removal (which proved to 
be very useful in case 2), its structural rigidity and, in case of malignant disease, resistance to infiltrative growth by tumour. Disadvantages include a greater tendency to displace, and the risk of obstruction by dried airway secretions. Gianturco $\mathrm{Z}$ stents do not move and probably do not induce drying of secretions; furthermore, they allow airflow through the stent itself - for example, when placed over the right upper lobe bronchus. Both types of stents probably could have been used in our patients. We preferred the silicone Dumon stents because of their structural rigidity, the ease by which they can be removed, and our greater experience with them.

We conclude that an endoscopically inserted silicone endoprosthesis may be a valid alternative to surgery for tracheal obstruction due to intrathoracic goitre in patients in whom surgery represents an unacceptable risk.

1 Hetzel MR, Smith SGT. Endoscopic palliation of tracheobronchial malignancies. Thorax 1991;46 325-33.

2 Colt HG, Dumon JF. Tracheobronchial stents: indications and applications. Lung Cancer 1993;9:301-6.

3 Dumon JF, Corsini A. Cicatriceal tracheostenosis. In: Bnnchoscopic Laser Resection Manual. Marseilles, France: 1989: 137.

4 Ingbar DH. The respiratory system in thyrotoxicosis. In: Braverman LE, Otiger RD, eds. The thymid. 6th edn Braverman LE, Otiger RD, eds. The

5 Calcaterra TC, Maceri DR. Aerodigestive dysfunction secondary to thyroid tumors. Laryngoscope 1981;91:701-7. 6 Alfonso A, Christoudias A, Amarrudin Q. Tracheal or eosophagal compression due to benign thyroid disease. Am f Surg 1981;142:350-4.

7 Karbowitz SR, Edelman LB, Nath S. Spectrum of advanced upper airway obstruction due to goitres. Chest 1985;87: 18-21.

8 Simonds AK, Dirving JD, Clarke SW, Dick R. Use of expandable metal stents in the treatment of bronchial obstruction. Thorax 1989;44:680-1.

\section{Massive haemothorax secondary to angiosarcoma}

\author{
A J McCleary
}

Cardiothoracic Surgery, Killingbeck

Hospital, Leeds, UK A J McCleary

Reprint requests to: A J McCleary,

10 Skippon Terrace,

Leeds LS14 3HA, UK

Received 5 August 1993 Returned to authors 15 October 1993

Revised version received

10 November 1993

Accepted for publication

5 January 1994

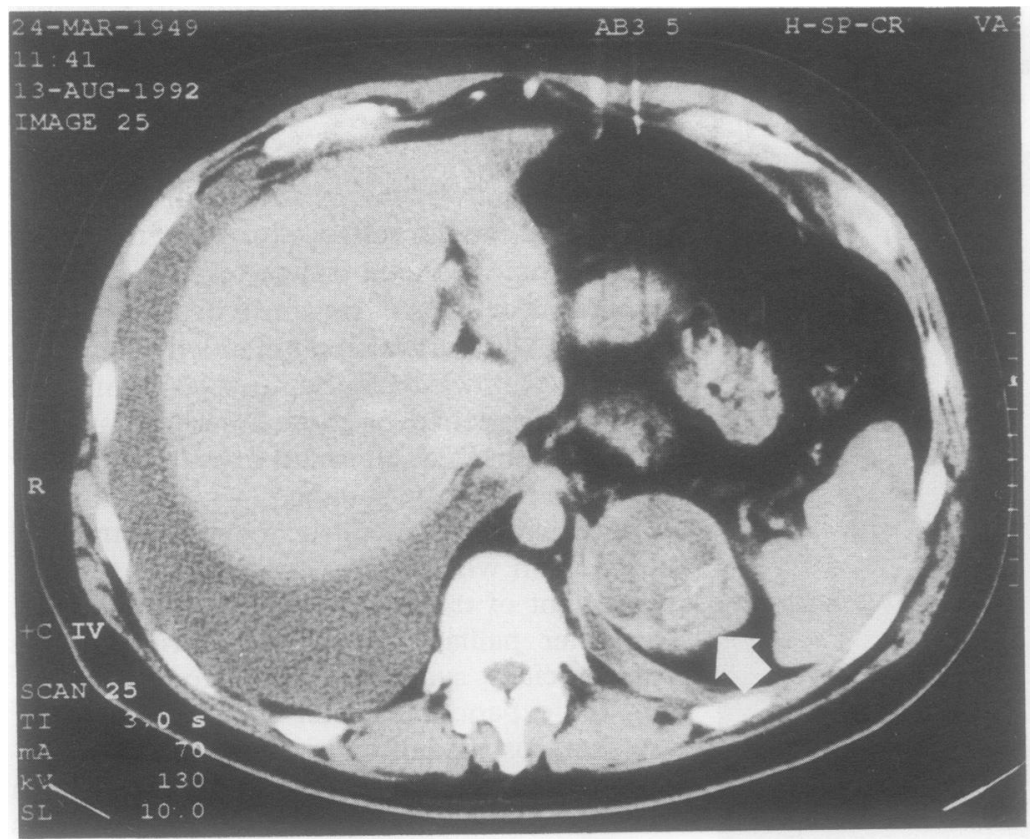

Figure 1 Computed tomographic scan of the abdomen showing a large mass in the left adrenal gland and right sided haemothorax.

\begin{abstract}
A patient who presented with recurrent haemoptyses was found to have an angiosarcoma of the adrenal gland which disseminated throughout the pleural space.
\end{abstract}

(Thorax 1994;49:1036-1037) abdomen revealed a left adrenal mass measuring $5 \times 4 \times 4 \mathrm{~cm}$ (fig 1 ).

A further 20 litres of blood were drained over the following five days. Clotting screens were normal and the adrenal tumour appeared to be non-functioning (urinary levels of 17ketosteroids and vanillylmandelic acid were normal). Pulmonary angiography and selective angiography of the intercostal arteries failed to demonstrate a definite bleeding point.

On the sixth day adrenalectomy and exploratory thoracotomy were performed. The adrenal mass was a well circumscribed cystic lesion filled with organising blood clot. Two litres of blood were removed from the left hemithorax to reveal visceral and parietal pleura covered in organising blood clot and studded with multiple haemorrhagic cysts $5-30 \mathrm{~mm}$ in diameter. The lung parenchyma appeared to be normal. Frozen sections of the lesions were reported as angiosarcoma. Bleeding was controlled and the chest closed.

Postoperatively the patient had a dense right sided hemiplegia. However, he remained stable 